\title{
Mutant SOD1 detoxification mechanisms in intact single cells
}

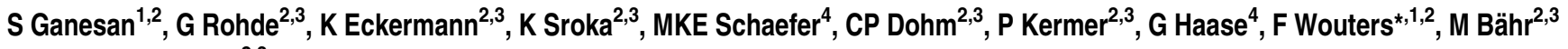 \\ and $\mathrm{JH}$ Weishaupt ${ }^{*, 2,3}$
}

Mutant superoxide dismutase 1 (mtSOD1) causes dominantly inherited amyotrophic lateral sclerosis (ALS). The mechanism for $\mathrm{mtSOD1}$ toxicity remains unknown. Two main hypotheses are the impairment of proteasomal function and chaperone depletion by misfolded mtSOD1. Here, we employed FRET/FLIM and biosensor imaging to quantitatively localize ubiquitination, as well as chaperone binding of mtSOD1, and to assess their effect on proteasomal and protein folding activities. We found large differences in ubiquitination and chaperone interaction levels for wild-type (wt) SOD1 versus mtSOD1 in intact single cells. Moreover, SOD1 ubiquitination levels differ between proteasomal structures and cytoplasmic material. Hsp70 binding and ubiquitination of wt and mtSOD1 species are highly correlated, demonstrating the coupled upregulation of both cellular detoxification mechanisms upon mtSOD1 expression. Biosensor imaging in single cells revealed that mtSOD1 expression alters cellular protein folding activity but not proteasomal function in the neuronal cell line examined. Our results provide the first cellby-cell-analysis of SOD1 ubiquitination and chaperone interaction. Moreover, our study opens new methodological avenues for cell biological research on ALS.

Cell Death and Differentiation (2008) 15, 312-321; doi:10.1038/sj.cdd.4402262; published online 9 November 2007

Amyotrophic lateral sclerosis (ALS) is a progressive fatal neurodegenerative disease caused by the selective loss of motoneurons. ${ }^{1}$ While ALS mostly occurs sporadically, 5-10\% of cases are hereditary. About $20 \%$ of familial and few sporadic cases show point or frameshift mutations within the superoxide dismutase 1 (SOD1) gene. ${ }^{2}$ The principle underlying mutant superoxide dismutase 1 (mtSOD1) toxicity seems to be a toxic gain - rather than loss - of function, since symptomatic mtSOD1-transgenic mice still express wild-type (wt), SOD1, some SOD1 mutants retain their enzymatic activity, ${ }^{2,3}$ and neither knockout of endogenous SOD1 nor transgenic overexpression of wtSOD1 affect the course of disease in mtSOD1-transgenic mice. ${ }^{4}$ Nonetheless, the downstream events in mtSOD1-mediated motoneuron death have not yet been resolved, although several hypotheses have been proposed that are based on properties that are specific for mtSOD1: altered protein folding, decreased solubility, and the propensity to form cytoplasmic aggregates. ${ }^{5-10}$ One of the major hypotheses states that misfolded mtSOD1, through binding, depletes neuroprotective chaperones. ${ }^{5-7}$ Accordingly, recombinant mtSOD1 inhibited chaperone activity in vitro. ${ }^{11}$ Moreover, expression of Hsp70, Hsp27, or Hsp40 decreased aggregation of mtSOD1 and protected against mtSOD1 toxicity in cell lines and primary motoneurons. ${ }^{6,12}$ However, overexpression of $\mathrm{Hsp} 70$ alone did not improve motoneuron survival in mtSOD1-transgenic mice. ${ }^{13}$ This is possibly explained by a requirement, in vivo, for correction of further cellular functions or the combined action of different chaperones in the prevention of motoneuron cell death. Indeed, arimoclomol treatment, known to upregulate both Hsp70 and Hsp90 in the spinal cord, extended the lifespan of SOD1 ${ }^{\text {G93A }}$-transgenic mice. ${ }^{14}$

Alternatively, proteasomal overload and dysfunction caused by the expression of aberrantly folded SOD1 might contribute to motoneuronal demise. ${ }^{15} \mathrm{mtSOD} 1$ is polyubiquitinated, can be degraded by the proteasome, ${ }^{16-18}$ and proteasomal activity was decreased in the lumbar spinal cord of SOD1 ${ }^{\text {G93A }}$-transgenic mice. ${ }^{19}$

SOD1 protein interactions, SOD1 ubiquitination, and altered cellular chaperone or proteasomal activities have been investigated previously before using biochemical assays on homogenized cells or subcellular fractions. ${ }^{5,11,19}$ A major drawback of these approaches is their disruptive nature and the lack of spatial information.

We made use of Förster resonance energy transfer (FRET), measured by fluorescence lifetime imaging (FLIM) to explore the mechanisms of mtSOD1 turnover and toxicity. The intrinsically quantitative nature of FLIM measurements allowed us to perform analytical biochemical measurements inside single cells. Using a recently developed FRET assay

${ }^{1}$ European Neuroscience Institute, Cell Biophysics Group, Waldweg 33, Göttingen 37073, Germany; ${ }^{2}$ DFG Research Center for Molecular Physiology of the Brain (CMPB), Göttingen, Germany; ${ }^{3}$ Department of Neurology, University of Göttingen, Waldweg 33, Göttingen 37073, Germany and ${ }^{4}$ Université de la Méditerranée, INSERM, Institut de Neurobiologie de la Méditerranée, Equipe AVENIR, campus de Luminy, Marseille cedex 09 F-13288, France

${ }^{*}$ Corresponding authors: F Wouters, Laboratory for Molecular and Cellular Systems, Department of Neuro- and Sensory Physiology, Humboldt-Allee 23, Göttingen 37073, Germany. Tel: + 4955139 12368; E-mail: fred.wouters@ gwdg.de or JH Weishaupt, Department of Neurology, University Hospital Göttingen, Robert-KochStrasse 40, Göttingen 37075, Germany. Tel: + 4955139 14343; Fax: + 4955139 14302; E-mail: jweisha@gwdg.de

Keywords: ALS; SOD1; FRET; chaperones; proteasome

Abbreviations: ALS, amyotrophic lateral sclerosis; AMS, 4-acetamide-4'-maleimidylstilbene-2,2'-disulfonic acid; CCS, copper chaperone for SOD1; cdYFP, conformational deficient yellow fluorescent protein; FLIM, fluorescence lifetime imaging; FRET, Förster resonance energy transfer; mtSOD1, mutant superoxide dismutase 1; REACh, resonance energy-accepting chromoprotein; SOD1, superoxide dismutase 1

Received 19.9.06; revised 03.8.07; accepted 11.9.07; Edited by L Greene; published online 09.11.07 
with improved sensitivity, ${ }^{20}$ we visualized SOD1 ubiquitination and chaperone interaction in intact single cells, and uncovered profound differences between wild-type and mtSOD1. Using innovative fluorescence-imaging-based biosensors, ${ }^{21}$ we also demonstrate in a cell-by-cell investigation differences with regard to chaperone activity between wt and mtSOD1transfected cells. Our findings substantially advance the understanding of cellular alterations by mtSOD1 and provide support for the involvement of altered chaperone function, rather than proteasomal deficits, in the mechanism of mtSOD1 toxicity.

\section{Results}

FRET/FLIM-based measurement of SOD1 ubiquitination using the REACh FRET acceptor. A recently developed FRET/FLIM protocol using a mutant non-fluorescent enhanced yellow fluorescent protein (EYFP) called resonance energy-accepting chromoprotein (REACh) conjugated to ubiquitin as FRET acceptor, and wt/mtSODEGFP as donor ${ }^{20}$; see also Materials and Methods) was used to study SOD1 ubiquitination distributions in intact neuronal cells. The spectral advantages of this new FRET pair over commonly used pairs render it highly efficient for the detection of FRET.

We used neuronal CSM14.1 cells for our experiments because of their robust and reproducible transfection efficiency that we require for our FLIM measurements and chaperone activity biosensor (see below). Importantly, their abundant cytoplasm allows detailed observation of the subcellular FRET distribution.

We verified that the EGFP tag on SOD1 does not profoundly alter the normal properties and maturation of the protein. To this end, we used an in-gel enzymatic SOD1 activity assay. ${ }^{22}$ We show that EGFP-SOD1 fusion proteins (wt and the 'wt-like' G93A mutant) form dimers and exhibit the expected superoxide dismutase activity (Figure $1 a$ and b). In contrast to EGFP-SOD1 ${ }^{\text {G93A }}$, enzymatic activity was lost by EGFP-SOD1 ${ }^{\text {G85R }}$, as observed before for the untagged SOD1 ${ }^{\text {G85R }}$ mutant. $^{23}$ The finding that our SOD1 fusion proteins still dimerize and - if not abolished by the respective mutation - exhibit normal dismutase activity, indicates that the EGFP tag does not preclude proper maturation, dimerization and copper loading of SOD1. This was further confirmed by the presence of the intramolecular disulfide bond in EGFP. SOD1 fusion proteins that is essential for activity and stability (Figure 1c). The copper chaperone for SOD1 (CCS) both inserts the catalytic copper cofactor and catalyzes the formation of the disulfide bond, ${ }^{24}$ thus also indicating proper recognition and processing of the protein by the CCS.

Cells expressing only EGFP-SOD1 exhibited typical EGFP lifetimes, independent of fusion of wt or mtSOD1 (2.0-2.2 ns; Figure $2 \mathrm{a}-\mathrm{c}$ ). Moreover, there is no evidence for EGFP ubiquitination, as fluorescence lifetimes in EGFP expressing cells are unaffected by coexpression of REACh-ubiquitin (Figure 2d).

Coexpression of wt or mutant EGFP-SOD1 with REAChubiquitin resulted in a strongly reduced lifetime. In some cytoplasmic areas, the lifetime dropped to approximately a

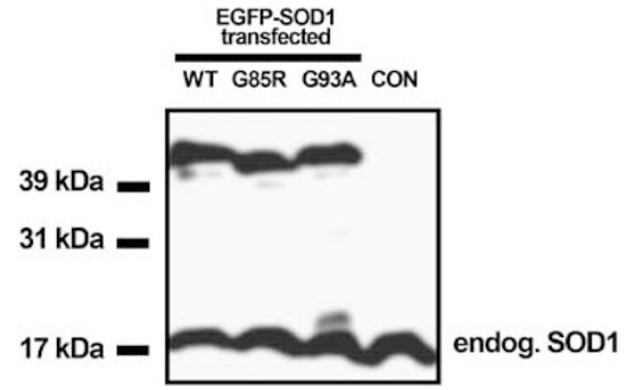

b
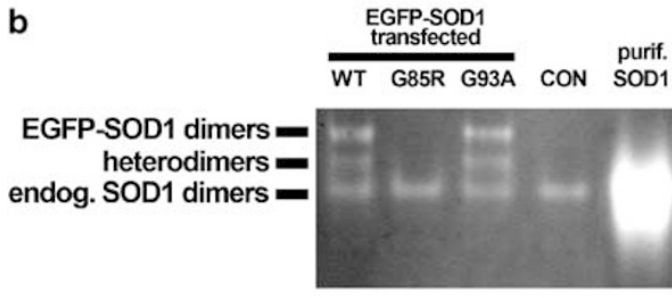

C

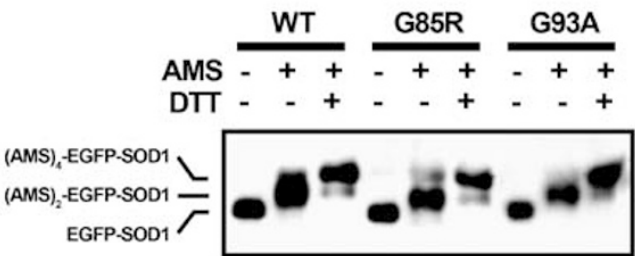

Figure 1 EGFP-SOD1 fusion proteins dimerize, are enzymatically active and form an intramolecular disulfide bond. The Western blot in (a) shows expression of EGFP-SOD1 fusion proteins in CSM14.1 cells $18 \mathrm{~h}$ after transfection. A 20- $\mu \mathrm{g}$ weight of protein lysate was loaded per lane and separated on denaturing SDS gel. Monomeric EGFP-SOD1 and endogenous wtSOD1 are stained using an anti-SOD1 primary antibody. (b) Shows SOD1 in the in-gel-zymography of lysates from EGFPSOD1-transfected CSM14.1 cells ( $20 \mu \mathrm{g}$ per lane). A $4-\mu \mathrm{g}$ weight of human wtSOD1 protein purified from erythrocytes was loaded as a positive control. Protein samples were separated on a non-denaturing polyacrylamide gel and stained for dismutase activity. Three distinct bands of SOD1 activity are observed in samples from EGFP-SOD $1^{\text {wt }}$ - and EGFP-SOD $1^{\mathrm{G} 93 \mathrm{~A}}$-transfected cells, representing the dimers of endogenous untagged SOD1, EGFP-SOD1 dimers, and the respective heterodimers. In contrast to the 'wt-like' SOD $1^{\mathrm{G} 93 \mathrm{~A}}$, in cells transfected with the dismutase-dead mtSOD1 ${ }^{\text {G85R }}{ }^{23}$ only endogenous SOD $1^{\text {wt }}$ activity could be detected. CON: untransfected control cells. The disulfide assay in (c) demonstrates efficient formation of the conserved intramolecular SOD1 disulfide bond that is catalyzed by CCS. AMS reacts with the two reduced thiol groups in SOD1 not involved in disulfide bond formation to (AMS) ${ }_{2}$-EGFP-SOD1, while the two other thiol groups form a disulfide bond and are not AMS reactive. This leads to a mobility shift on a Western blot using a denaturing gradient gel and an anti-SOD1 primary antibody (see also Materials and Methods section). Treatment with DTT for $1 \mathrm{~h}$ at $37^{\circ} \mathrm{C}$ reduces the disulfide bond, making two additional thiol rests available for reaction with AMS, as shown by a further mobility shift due to formation of $(\mathrm{AMS})_{4^{-}}$EGFP-SOD1

$1.2 \mathrm{~ns}$ (Figure $2 \mathrm{e}-\mathrm{j}$ ), indicating high FRET and a direct interaction of SOD1 with ubiquitin.

The extent and distribution of ubiquitination was clearly different between EGFP-fused mtSOD1 and wtSOD1. While fluorescence lifetime values only moderately decreased for EGFP-SOD1 ${ }^{\text {wt }}$ (Figure $2 \mathrm{e}$ and $\mathrm{f}$ ), they were markedly decreased for EGFP-mtSOD1 (Figure 2g-j) with higher ubiquitination levels for SOD1 ${ }^{\text {G85R }}$ (Figure $1 \mathrm{~g}$ and $\mathrm{h}$ ) than for SOD $1^{\text {G93A }}$ (Figure $2 \mathrm{i}$ and $\mathrm{j}$ ). Statistical analysis of cumulative fluorescence lifetime histograms (Figure 2k) confirmed the statistical significance of the differences (Figure 2l). 
Spots of high FRET were observed perinuclearly, strongly suggestive of proteasomes. This most likely reflects the dynamic accumulation of ubiquitinated material in the turnover of EGFP-SOD1 ${ }^{\text {wt }}$ due to protein overexpression (Figure $2 \mathrm{e}$ and f). The ubiquitination levels of SOD1 ${ }^{\text {G93A }}$ and SOD1 ${ }^{\text {G85R }}$ in the proteasome structures were significantly higher than for SOD $1^{\text {wt }}$ (Figure $2 \mathrm{~g}-\mathrm{i}$; quantitation in Figure 3). Surprisingly, while SOD1 ${ }^{\text {G85R }}$ showed a higher degree of overall ubiquitination than the SOD1 ${ }^{\text {G93A }}$, the degree of ubiquitination was identical for both mutants in proteasomal areas (Figure 3), suggesting their maximal absolute ubiquitination.

We also detected differences in the spatial distribution of SOD $1^{\text {G85R }}$ and SOD1 ${ }^{\text {G93A }}$ ubiquitination: most (75\%) EGFPSOD1 ${ }^{\text {G93A }}$ cells displayed the shortest lifetime (i.e., highest ubiquitination) in cytoplasmic aggregates (Figure $2 \mathrm{i}$ and j). In contrast, the same fraction of EGFP-SOD1 ${ }^{\text {G85R }}$ cells have higher ubiquitination outside aggregates, and reached maximum levels in the cell periphery where only non-aggregated, most likely soluble, EGFP-SOD ${ }^{\text {G85R }}$ is found (Figure $2 \mathrm{~g}$ and h). In fact, close-to-normal lifetimes were often observed inside large SOD1 ${ }^{\text {G85R }}$ aggregates (Figure $2 \mathrm{~g}$ and $\mathrm{h}$ ). No SOD1 ubiquitination was observed in nuclear regions. Quantification of proteasome function in single cells
expressing SOD1. We confirmed that CSM14.1 cells are susceptible to mtSOD1 toxicity (Supplementary Figure) as with other neuronal cell types. ${ }^{12,25}$ The increased and spatially distinct ubiquitination of EGFP-mtSOD1 prompted us to test the proteasomal overload hypothesis as a mtSOD1 toxicity mechanism. In order to find evidence for altered proteasome function in intact single cells expressing mtSOD1, we utilized a destabilized ECFP containing a PEST sequence (ECFP-PEST) for degradation targeting and, consequently, rapid turnover $\left(t_{1 / 2}= \pm 2 \mathrm{~h}^{26}\right)$. Transfection of ECFP-PEST results in negligible steadystate levels and only faint ECFP fluorescence, unless proteasomal degradation is impaired ${ }^{26}$; Figure $4 a$ and b). A concentration reference was provided by coexpression of (non-destabilized) EYFP to standardize ECFP-PEST fluorescence against concentration differences. Division of ECFP by EYFP fluorescence gives the proteasomal stability metric. Treatment with the proteasome inhibitor lactacystin at a concentration of $20 \mu \mathrm{M}$ for $24 \mathrm{~h}$ resulted in a $\sim 6$-fold reduction in proteasomal activity, confirming the validity of our assay (Figure $4 a$ and b). Quantification of ECFP-PEST stability did not reveal any changes in proteasomal activity between cells transfected with control vector, wt or mtSOD1 for $24 \mathrm{~h}$ (data not shown) or even $48 \mathrm{~h}$ (Figure $4 \mathrm{a}$ and $\mathrm{b}$ ).

Interaction of SOD1 with Hsp70. In the absence of proteasomal dysfunction, chaperone depletion by mtSOD1 remains as an alternative explanation for the impairment of neuronal viability. We employed the FRET/FLIM technique to study the interaction of SOD1 (wt and mutant) with the Hsp70 chaperone in single cells. Again, EGFP-SOD1 served as FRET donor, and Hsp70 was fused to REACh acceptor (REACh-Hsp70). As before, the expected unperturbed EGFP lifetimes were obtained for wt and mtSOD1 fusions (Figure $5 a-c)$. In contrast, coexpression of REACh-Hsp70 with EGFP-SOD $1^{\text {wt }}$ mildly reduced the EGFP lifetime (to about $1.7 \mathrm{~ns}$; Figure $5 \mathrm{~d}$ ), indicating that SOD1 ${ }^{\text {wt }}$ interacts with Hsp70. This interaction was clearly increased for EGFPSOD1 $1^{\text {G85R }}$ and EGFP-SOD1 ${ }^{\text {G93A }}$, as shorter lifetimes were measured, approaching $1.2 \mathrm{~ns}$ in some subcellular areas (Figure $5 \mathrm{e}$ and $\mathrm{f}$ ). Figure $5 \mathrm{~g}$ and $\mathrm{h}$ show the respective quantitative analysis, with $\mathrm{SOD} 1^{\mathrm{G} 85 \mathrm{R}}$ interacting more intensively than SOD1 ${ }^{\text {G93A }}$.

Compared with the ubiquitination of SOD1 described above, the distribution of SOD1/Hsp70 interactions appeared more homogeneously, without appreciable differences between aggregates and cytoplasmic, soluble EGFP-SOD1. The shortest fluorescence lifetimes were observed in the cell periphery.

Even though EGFP-SOD $1^{\text {wt }}$ clearly entered the nucleus, no interaction with Hsp70 was measured in this compartment, providing an intrinsic biological control for the specificity and relevance of the found interactions. Notably, coexpression of $\mathrm{REACH}-\mathrm{Hsp} 70$ altered the appearance of EGFP-mtSOD1 (right panels in Figure $5 e$ and $\mathrm{f}$ ); the mtSOD1 accumulations appeared less confined and with smoother borders (compare also with Figure $2 a-c)$. Hsp70 overexpression also resulted in a lower density of EGFP-mtSOD1 aggregates, suggesting that the chaperone improved the solubility of the protein.

Hsp70 interaction of SOD1 mutants correlates with
ubiquitination. Quantitative cell-by-cell FLIM analysis of
ubiquitination and Hsp70 binding of wt and mtSOD1 allowed
us to correlate both detoxification mechanisms. Correlation
of the data sets from wtSOD1, G93A, and G85R mtSOD1
revealed a close relationship ( $r=0.97$; Figure 6$)$ between
both events. It thus appears that the increased cellular
toxicity of SOD1 species increases both ubiquitination and

\footnotetext{
Figure 2 Quantification and localization of SOD1 ubiquitination in intact neuronal cells measured by FLIM. (a-j) Representative fluorescence lifetime images (fluorescence lifetime in color coding, left panels) and distribution of the EGFP donor constructs (EGFP intensity pictures, right panels). The scale bar in (a) indicates the color-coding of fluorescence lifetime values. Warmer colors indicate lower lifetimes, that is, higher FRET efficiencies, and cooler colors indicate higher lifetimes, that is, lower FRET efficiencies. (a-d) Typical EGFP fluorescence lifetimes (1.8-2 ns) were observed in CSM14.1 cells expressing the EGFP-SOD1 donor constructs only (a-c), or in cells coexpressing EGFP and REACH-ubiquitin (d). Coexpression of EGFP-SOD1 constructs with REACh-ubiquitin resulted in strongly shortened lifetimes (e-j), with a regionally heterogenous distribution. $(\mathbf{f}, \mathbf{h}, \mathbf{j})$ These panels show the magnifications of the representative cells shown in $(\mathbf{e}, \mathbf{g}, \mathbf{i})$. (k) Shows the cumulative histograms of FRET efficiencies of wt and mutant EGFP-SOD1 when coexpressed with REACH-ubiquitin. Lifetime values were converted to FRET efficiencies by comparison to the EGFP lifetime of control cells expressing EGFP-SOD1 ${ }^{\text {wt }}$ in the absence of REACh-labeled interaction partner (REACH-ubiquitin). (I) Shows the quantification of histograms shown in (k). The cumulative distribution of the FRET efficiencies of control cells expressing EGFP-SOD1 without coexpression of the REACh-labeled ubiquitin were fitted with a Gaussian function to obtain the average plus three times standard deviation value $(3 \sigma)$. Any values exceeding this threshold thus represent increases that deviate from the control situation. The probability of obtaining significant FRET was derived for each cell by integration of the normalized FRET distribution histograms above the $3 \sigma$ threshold (I). CSM14.1 cells (from three independent experiments) were imaged $18 \mathrm{~h}$ after transfection with the respective expression constructs. Data sets are presented as mean \pm S.E.M. Statistical analysis was performed by ANOVA and Tukey's post hoc analysis. PDF, probability distribution function; Ubq, ubiquitin
} 
Hsp70 binding in parallel, strongly suggesting that both detoxification pathways are upregulated in an attempt to reduce the amount of insoluble, aggregation-prone, toxic SOD1 protein.

Functional effect of mtSOD1 on cellular chaperone activity. As we found increased chaperone binding by mtSOD1, we investigated its functional influence for cellular chaperone activity in the single cell using a recently developed fluorescence imaging-based, broad-specificity chaperone activity biosensor. $^{21}$ This biosensor consists of a folding-impaired mutant of yellow fluorescent protein (cdYFP) tagged with an HA epitope. Its folding and maturation that is necessary for the generation of the chromophore is impaired and thus only minor fluorescence is generated. However, in cells experiencing increased a

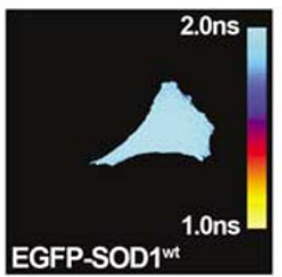

C

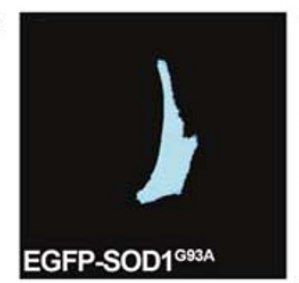

e

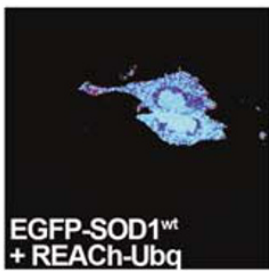

g

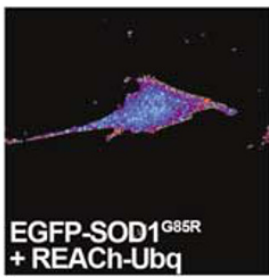

i

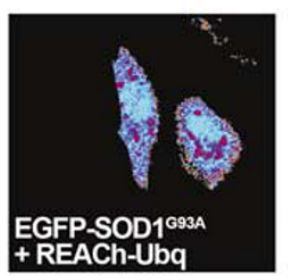

$\mathbf{k}$

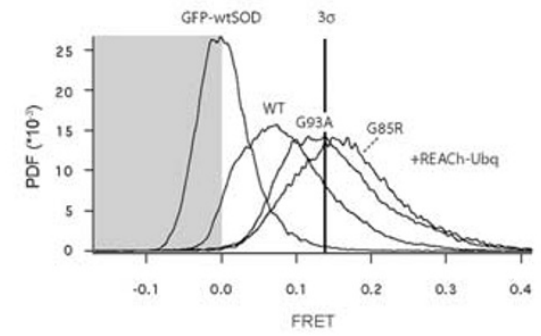

b

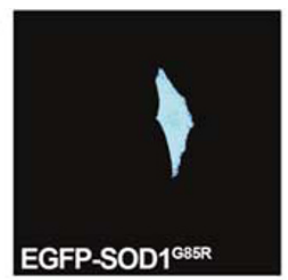

d

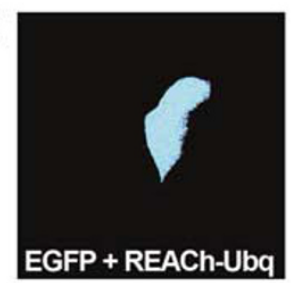

f

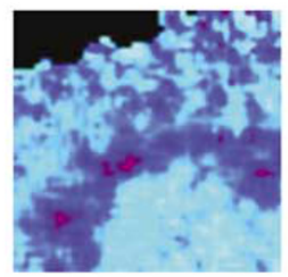

h

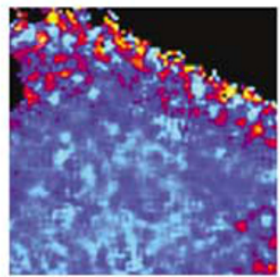

j

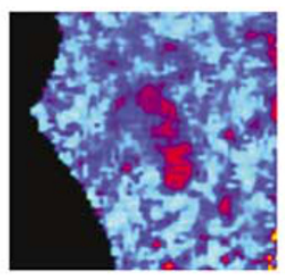

I

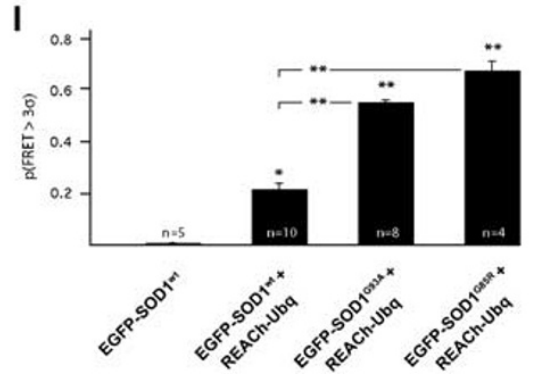

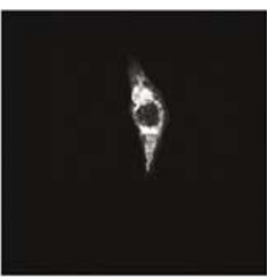
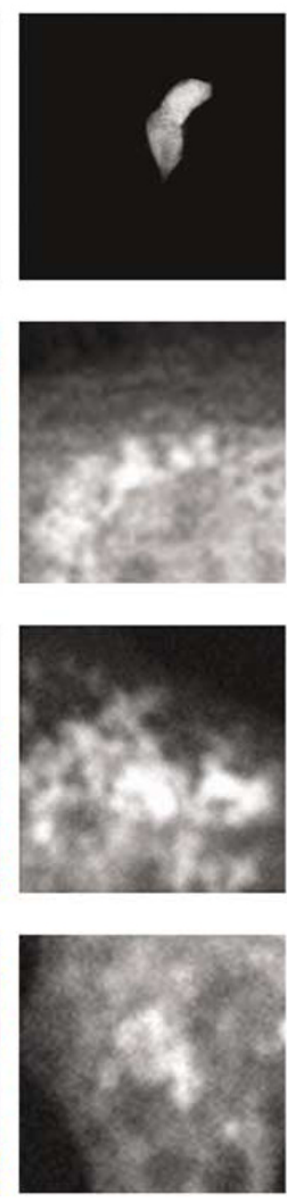

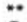




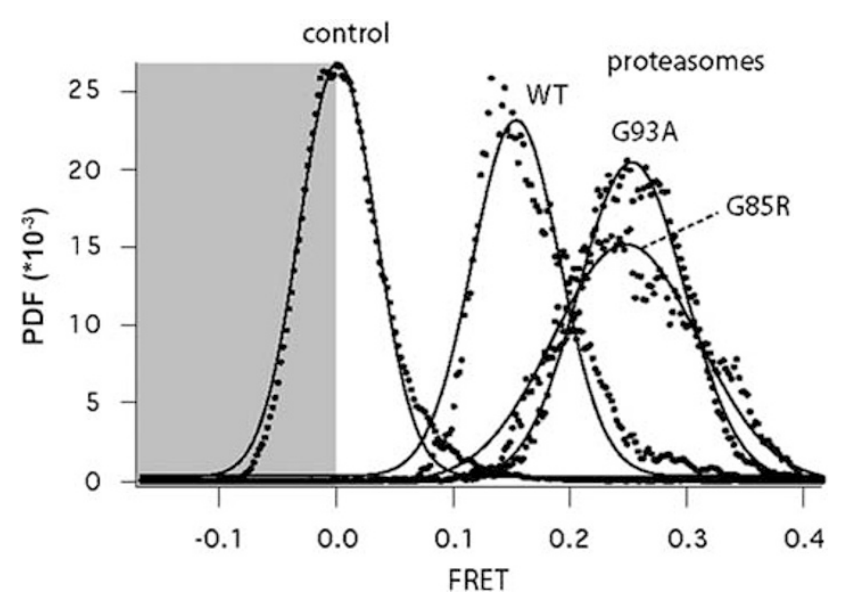

Figure 3 Cumulative histograms of wt and mutant EGFP-SOD1/REACHubiquitin FRET efficiencies in proteasomal areas. Lifetime values were converted to FRET efficiencies by comparison to the EGFP lifetime cells expressing EGFP. SOD1 in the absence of the labeled interaction partner REACH-ubiquitin (control). The cumulative FRET efficiencies ( $x$-axis) for the different proteins are presented as in Figure 2. CSM14.1 cells were imaged $18 \mathrm{~h}$ after transfection with the respective expression constructs. Proteasomes were defined as perinuclear punctate structures with high FRET between EGFP-SOD1 ( $w t$, G85R, and G93A mutants) and REACh-ubiquitin and were segmented for processing by thresholding of the FRET efficiencies. Data sets are presented as mean \pm S.E.M. Statistical analysis was performed by ANOVA and Tukey's post hoc analysis. PDF, probability distribution function

chaperone activity, cdYFP conformation can recover to yield fluorescence similar to native YFP. Expression levels of the cdYFP reporter are normalized to the inert HA immunostaining signal (see also Materials and Methods).

The chaperone activity in the cytosol increased upon coexpression of wtSOD1, approaching the levels reached by ECFP-Hsp70 overexpression, which was used as a positive control (Figure 7a-d).

Coexpression of SOD $1^{\text {G85R }}$ also induced chaperone activity, but significantly less than with wtSOD1 (Figure 7a-d). Chaperone activity in SOD1 ${ }^{\mathrm{G} 93 \mathrm{~A}}$-expressing cells even dropped below baseline values obtained in control cells expressing only cdYFP (Figure 7a-d).

\section{Discussion}

With the use of genetically encoded optical biosensors for proteasomal and chaperone activity, Hsp70/SOD1 interaction, and SOD1 ubiquitination, we investigated the cellular detoxification pathways that are elicited by the expression of mtSOD1. We demonstrate profound differences in chaperone binding and activation, as well as ubiquitination between wt and ALS-associated mtSOD1.

SOD1 ubiquitination was quantitatively imaged in intact single cells using the non-fluorescent REACh acceptor for FRET with EGFP. ${ }^{20}$ In contrast to biochemical methods, our FLIM/FRET measurements allow the spatial quantification of SOD1 ubiquitination and its heterogeneity between cells. Overall, mtSOD1 ubiquitination is enhanced compared with wtSOD1. Also in proteasomal areas, mtSOD1 ubiquitination is clearly higher than for wtSOD1. However, in contrast to cytoplasmic regions, levels are identical for $\operatorname{SOD} 1^{\mathrm{G} 93 \mathrm{~A}}$ and

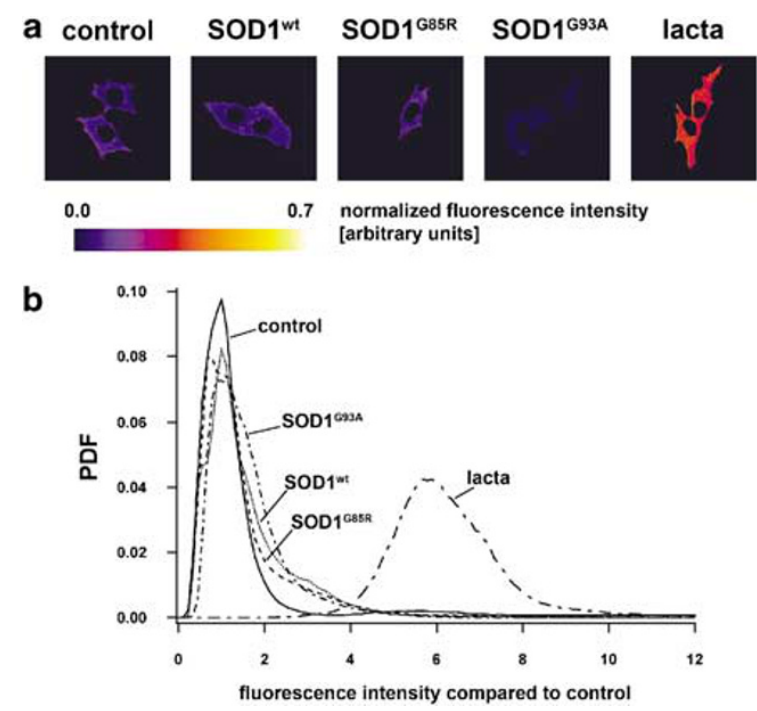

Figure 4 Overexpression of SOD1 does not impair proteasomal function in CSM14.1 cells. The proteasomal substrate destabilized enhanced cyan fluorescent protein, containing a PEST amino-acid sequence (ECFP-PEST) that targets the ECFP protein for degradation, was used to measure proteasomal function in neuronal CSM14.1 cells by relating its fluorescence ( concentration) to coexpressed EYFP. ECFP-PEST/YFP ratios (i.e., normalized ECFP fluorescence intensity) during coexpression of the respective SOD1 construct, or treatment with lactacystin ( $20 \mu \mathrm{M}$ for $24 \mathrm{~h}$ ) as a positive control, are indicated in (a) (color coded as indicated by the scale bar). (b) Shows the cumulative histograms summarizing data from 8-12 cells per condition, showing that SOD1 overexpression does not change ECFP-PEST levels, which, however, are greatly enhanced by lactacystin. Values are normalized to the control situation without expression of SOD1 protein (transfection of empty vector). The expression of the respective SOD1 proteins was verified by immunostaining of the myc epitope tag contained in the constructs (Cy5coupled secondary antibody) for each cell included in the analysis. PDF, probability distribution function; Lacta, lactacystin

SOD $1^{\mathrm{G} 85 \mathrm{R}}$, suggesting increased but saturated ubiquitination levels for the mutant species in proteasomal areas. This either reflects more extensive polyubiquitination (i.e., decreased deubiquitination) or additional 'pathological' ubiquitination sites in mtSOD1.

Our results on SOD1 ubiquitination are in agreement with strong biochemical evidence that SOD1 is at least in part degraded by an ubiquitin-dependent proteasomal pathway. ${ }^{16-18}$ Moreover, the discovery of ubiquitin ligases specifically ubiquitinating $m \mathrm{mSOD} 1^{27,28}$ may relate to our observation of enhanced ubiquitination of mtSOD1. Nevertheless, it is likely that additional degradation pathways other than ubiquitin-proteasomal degradation participate in SOD1 turnover. For example, a recent paper shows that macroautophagy and proteasomal degradation contribute approximately equally to SOD1 clearance. ${ }^{16}$ Furthermore, results from cell-free assays suggest that the $20 \mathrm{~S}$ proteasome preferentially degrades metal-depleted, monomeric mtSOD1 also in a ubiquitin-independent manner. ${ }^{29}$ Whether the holoenzyme or the demetallated, S-S bond reduced form of SOD1 is preferentially ubiquitinated and targeted for degradation can, as yet, not be determined by our approach.

Accumulating evidence suggests a triage decision in handling toxic misfolded proteins in aggregopathies; proteins are either (1) successfully refolded, (2) stored in aggregates, 


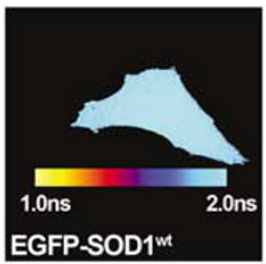

b

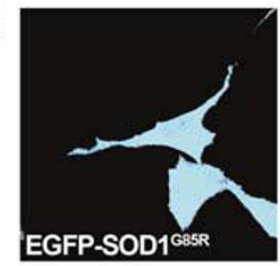

C

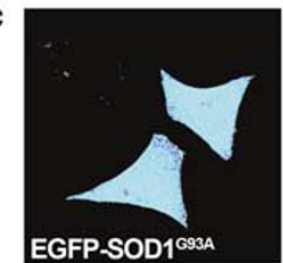

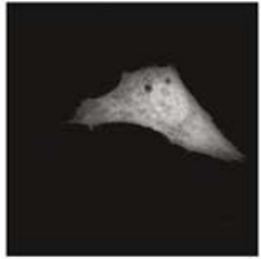
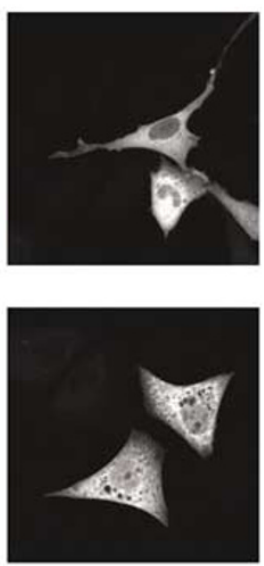

g

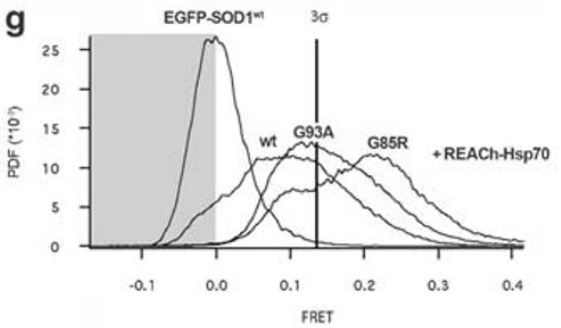

d
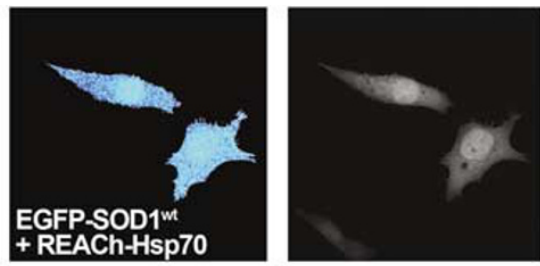

e
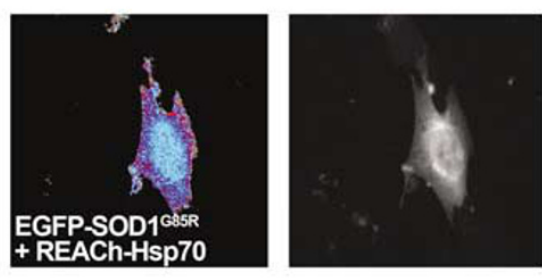

f

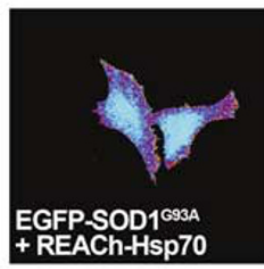

h

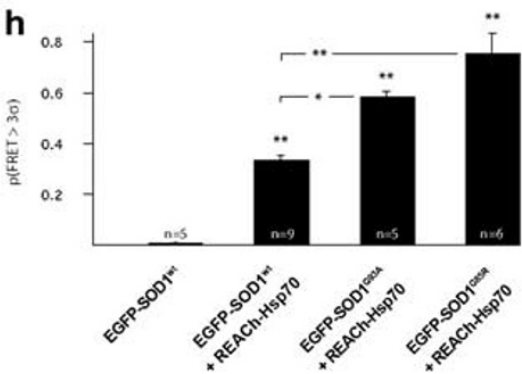

Figure 5 Quantification and localization of the interaction between SOD1 and Hsp70 in intact neuronal cells by FLIM. (a-f) Representative fluorescence lifetime images (fluorescence lifetime color coded, left panels) and distribution of the EGFP donor constructs (EGFP intensity pictures, right panels). The scale bar in (a) indicates the colorcoding of fluorescence lifetime values. (a-c) Typical EGFP fluorescence lifetimes were observed in CSM14.1 cells expressing only the EGFP-SOD1 donor constructs. Coexpression of wt EGFP-SOD1 constructs with Hsp70-REACh resulted in mildly reduced lifetime values (d), while strongly enhanced FRET was observed when Hsp70REACh was coexpressed with mutant EGFP-SOD1 constructs $(\mathbf{e}, \mathbf{f})$. (g) Shows the cumulative histograms of FRET efficiencies; panel (h) provides the respective quantitative analysis of the histograms as in Figure $2 \mathrm{l}$ and described in the Materials and Methods section. CSM14.1 cells were imaged $18 \mathrm{~h}$ after transfection with the respective expression constructs. Data sets are presented as mean \pm S.E.M. Statistical analysis was performed by ANOVA and Tukey's post hoc analysis. PDF, probability distribution function

or (3) targeted for proteasomal degradation or other degradation pathways (for review see reference McClellan et al. ${ }^{7}$ ). An imbalance between refolding and degradation may constitute an early event in cellular death, especially in neurodegenerative diseases where a number of proteins functionally link protein folding to proteasomal function. The close correlation between SOD1 ubiquitination and Hsp70 binding supports their causative interaction and parallel induction. Using the G85R and G93A SOD1 mutants, we exemplify species with preserved (SOD1 ${ }^{\mathrm{G} 93 \mathrm{~A}}$ ) or impaired (SOD1 ${ }^{\mathrm{G} 85 \mathrm{R}}$ ) enzymatic activity, and differences in the degree of protein misfolding and stability. ${ }^{2,3}$ In contrast to the cytoplasmic distribution of ubiquitinated SOD1 ${ }^{\text {G85R }}$, ubiquitinated SOD1 ${ }^{\text {G93A }}$ was enriched in SOD1 aggregates. We propose that these distinct ubiquitination patterns might explain their different toxicity and histopathology..$^{9,10}$

Despite the observed increased ubiquitination, mtSOD1 did not impair proteasomal function as measured by the steady- state levels of destabilized ECFP. In contrast, others could detect proteasomal impairment using fluorogenic proteolysis substrates in vitro. ${ }^{17,19}$ Our approach represents a global proteasomal measurement that may miss subtle changes in the subsets of proteasomal activities. On the other hand, biochemical methods estimate proteasomal function in protein lysates far from the cellular physiological conditions. Moreover, a presymptomatic decrease in proteasomal activity was detected in the lumbar but not cervical spinal cord of SOD $1^{\text {G93A }}$ transgenic mice. ${ }^{19}$ Thus, although our findings show that protein folding and the ubiquitination machinery are involved in the cellular response to mtSOD1, we could not substantiate the hypothesis of 'proteasomal overload' as a main contributor to toxicity. This could be explained by the occurrence of (mono-) ubiquitination, which does not always result in proteasomal degradation, since our assay does not distinguish between mono- and polyubiquitination. Moreover, as outlined above, additional pathways besides 


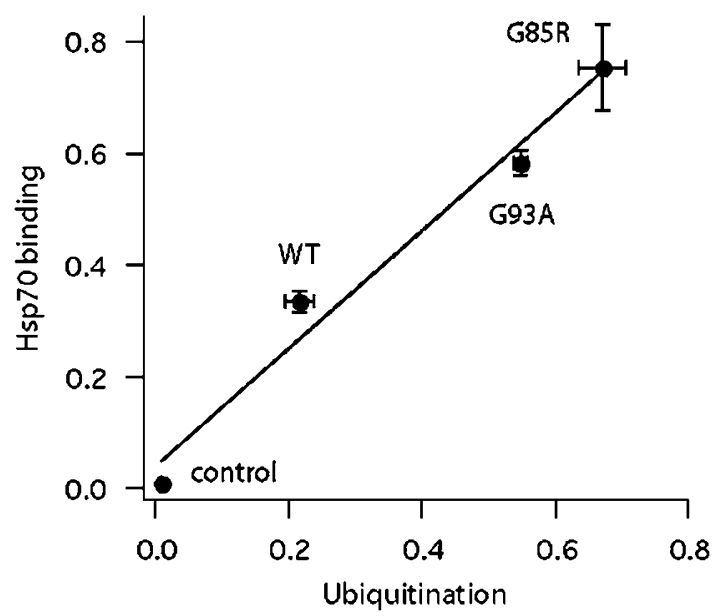

Figure 6 SOD1 ubiquitination positively correlates with $\mathrm{Hsp} 70$ binding in intact single cells. FRET efficiencies of EGFP-SOD1/REACh-Hsp70 binding were plotted against FRET efficiencies of EGFP-SOD1/REACh-ubiquitin experiments, and revealed a high and linear correlation between both parameters $(r=0.97)$. Data sets are presented as mean \pm S.E.M.

ubiquitin-dependent proteasomal degradation may contribute to SOD1 degradation, possibly enabling the cell to cope with an increased burden of misfolded SOD1 targeted for degradation.

Rather, we found evidence in favor of the depletion of cellular chaperones by mtSOD1, which was suggested before. $^{6,11}$ Shielding of toxic misfolded proteins by chaperone binding appears to be a cellular coping mechanism, which might fail when critical amounts of aggregation-prone proteins accumulate, specifically in neurons that do not 'dilute' toxic proteins during cell division. Hsp70 was recently shown to be included in $\alpha$-synuclein aggregates. ${ }^{30}$ This points to the possibility of cellular chaperone trapping during their futile attempt to dissolve $\alpha$-synuclein aggregates or to prevent their formation. The growing aggregate surface is shielded and rendered physiologically inert by chaperones, at the same time depleting the cells from their protective and physiological functions. In our case, a similar mechanism appears to be at work; chaperones engage in a 'deadly embrace' with mtSOD1 and their depletion leaves the toxic insult uncompensated, leading to cellular death. However, we could show that SOD1/ Hsp70 interaction primarily occurred outside aggregates, in accordance with the recently reported transient nature of Hsp70 interaction with aggregated mtSOD1. ${ }^{8}$ Hsp70 and other chaperones may thus be predominantly sequestered by mono- or oligomeric SOD1 species that are still soluble, rather than being trapped in 'end stage' SOD1 aggregates.

The increased binding of Hsp70 to mtSOD1 versus wtSOD1 implicates Hsp70 as a critical component in toxicity. However, as our chaperone biosensor does not distinguish between different chaperones, other chaperones in addition to Hsp70 are likely involved.

A chaperone activity-enhancing property of wtSOD1 has not been described before, but is in line with its general protective role. It remains to be determined whether SOD1 directly modulates chaperone activity, or even possesses chaperone activity itself, as we did not detect an upregulation of Hsp70 or Hsp40 protein levels by wtSOD1 (not shown).
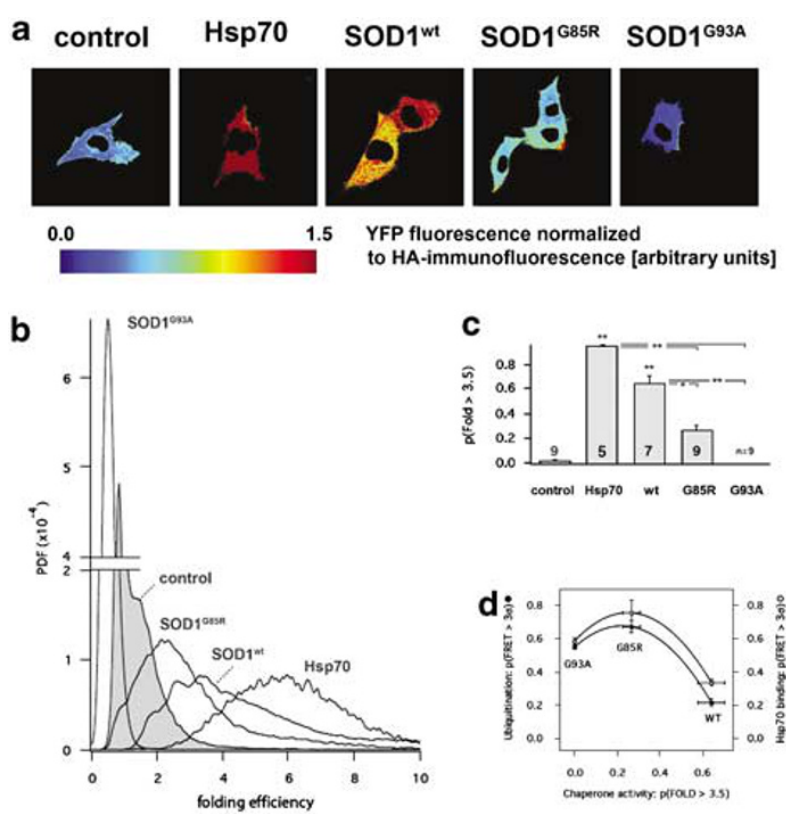

Figure 7 Overexpression of SOD1 alters cellular protein folding activity. The genetically encoded chaperone activity sensor cdYFP (see text and Materials and Methods section) was used to determine protein folding activity in intact single cells upon SOD1 expression. Expression levels of the cdYFP reporter are normalized by ratio imaging with the folding-independent HA immunostaining signal (Cy5-coupled secondary antibody). (a) Shows representative cells with the normalized colorcoded chaperone activity during expression of the indicated SOD1 variants. In one condition, ECFP-Hsp70 was coexpressed as a positive control. Respective cumulative histograms are shown in (b). (c) Depicts the results of the quantitative analysis (quantification of histograms performed as described in the figure legend for Figure 2l, and the Materials and Methods section). Fluorescence intensities were normalized to control cells expressing cdYFP only. SOD1 and Hsp70-ECFP constructs were expressed for $16 \mathrm{~h}$. In (d), protein folding activity under expression of the different SOD1 species is correlated with Hsp70 binding (white symbols) or ubiquitination (black symbols), respectively. PDF, probability distribution function

The proposed chaperone depletion by the mutants would match the widely assumed toxic gain of function of the SOD1 mutations. $^{2-4}$ Due to the decreased capacity of motoneurons to upregulate chaperones during/after cellular stress, ${ }^{31,32}$ the net effect of mtSOD1 on chaperone activity could be negative in motoneurons. In fact, the chaperone activity with SOD $^{\text {G93A }}$ expression was even below the control situation, in accordance with decreased protein folding activities in protein lysates from spinal cords of transgenic mice. ${ }^{11}$ However, at the current stage, we cannot exclude a loss of function effect concerning the observed chaperone enhancement by ALS-related mutations, and further work is required to verify the observed inhibitory effect of SOD1 mutants in motoneurons. In addition, the sum of mtSOD1 effects on protein folding activity might also differ between non-neuronal cell types. This could be of relevance as well, as it was shown that SOD1-related motoneuron death is not purely motoneuron-autonomous. ${ }^{33,34}$

Our observation that Hsp70 overexpression changed the appearance of SOD1 aggregates - suggesting their dispersal - further implies that increasing cellular chaperone activity may be useful for ALS treatment. Consistent with this hypothesis, overexpression of heat-shock proteins protected against mtSOD1 toxicity in experiments using cell lines or 
primary motoneurons. ${ }^{6,12}$ However, overexpression of one chaperone alone does not improve motoneuron survival in in vivo ALS models, ${ }^{13}$, possibly because correction of further cellular functions or the combined action of several different chaperons may be required before motoneuronal apoptosis is prevented in vivo. Indeed, treatment with arimoclomol, a compound that leads to increased levels of both Hsp70 and Hsp90 in the spinal cord, extended the lifespan of SOD1 ${ }^{\text {G93A }}$ transgenic mice. ${ }^{14}$

A technical caveat has to be mentioned, because the use of fluorescent protein constructs, widely used for most cell biological FRET experiments, generally implies the possibility that the fluorescent tag alters the properties of the fused protein. For example, the EGFP tag could reduce SOD1 copper loading by precluding the interaction with its copper chaperone, or abolish the formation of physiological SOD1 dimers. SOD1 holoenzyme and demetallated, disulfidereduced SOD1 may be degraded by different pathways. ${ }^{29} \mathrm{~A}$ potential shift in the cellular degradation pathway, for example, from lysosomal to proteasomal or from ubiquitindependent to ubiquitin-independent pathways, could then result in alterations and impairment of the cytoplasmic chaperone response. However, we showed that the EGFP. SOD1 fusion proteins form homodimers and heterodimers with endogenous untagged SOD1. Moreover, both dimers exhibited the expected SOD activity, in accordance with the robust enzymatic activity of previously published EGFP. tagged SOD1 fusion constructs. ${ }^{35}$ Finally, we could demonstrate the formation of the conserved intramolecular SOD1 disulfide bond in the fusion proteins. This indicates proper processing by CCS, which inserts the catalytic copper cofactor and catalyzes the formation of the conserved disulfide bond. ${ }^{24}$ Substantial disruption of the physiological maturation, function, and degradation of our EGFP-tagged SOD1 fusion proteins is thus very unlikely. It is important to note that whereas the physiological function of several other mutant proteins related to neurodegenerative diseases, for example, $\alpha$-synuclein or APP, is not even known, the function and maturation of fluorescent protein-SOD1 fusion constructs can be verified.

In summary, we performed the first comprehensive FRETand biosensor-based study on SOD1 ubiquitination and chaperone binding, as well as the activities of proteasomal degradation and protein folding, demonstrating profound differences between wt and mtSOD1. We show that central facets of cellular ALS pathogenesis, specifically the interaction of SOD1 with ubiquitin or chaperones, can be visualized in intact single cells and quantified with spatial resolution. Our results contribute to the understanding of mtSOD1 handling and toxic action in neuronal cells, and introduce new methodological avenues, in particular for the study of cell biology in ALS.

\section{Materials and Methods}

Chemical reagents. Cell culture reagents were purchased from PAA Laboratories. Lactacystin was obtained from Sigma-Aldrich (Cölbe, Germany).

Expression vectors. The cdYFP vector was produced by random mutagenesis as described previously. ${ }^{21}$ EGFP-SOD1 constructs were cloned by PCR amplification of the human wtSOD1 sequence and subsequent subcloning into a pcDNA3.1 expression vector (Invitrogen, Carlsbad, USA) C-terminally of the EGFP insert with Kpnl/Nott. Respective SOD1 mutant expression vectors were generated by PCR mutagenesis. The Pd2ECFP-N1 expression plasmid for PESTCFP (as proteasome activity sensor) and the EYFP expression vector were purchased from Clontech (Mountain View, CA, USA). REACh and REACh-ubiquitin expression constructs were described recently. ${ }^{20}$ REACh was cloned into the multiple cloning site of the pcDNA3.1 expression vector after PCR amplification using HindllI/BamHI. Rat Hsp70 was inserted C-terminally of REACh using $\mathrm{BamHI} / \mathrm{EcoRI}$.

Cell culture and transfection. The rat nigrostriatal cell line CSM14.1, immortalized by introduction of the temperature-sensitive simian virus 40 large $T$ antigen, ${ }^{36}$ was cultured in complete DMEM supplemented with $10 \%$ heatinactivated fetal calf serum, $100 \mathrm{U} / \mathrm{ml}$ penicillin $\mathrm{G}$, and $100 \mu \mathrm{g} / \mathrm{ml}$ streptomycin at $32{ }^{\circ} \mathrm{C}$ in $5 \% \mathrm{CO}_{2}$. For transfection, cells were seeded on poly-L-ornithine/laminin (Sigma, Cölbe, Germany)-coated coverslips (12 $\mathrm{mm}$ diameter) at a density of $15000 \mathrm{cells} / \mathrm{cm}^{2}$. Cells were then incubated with Lipofectamine 2000 according to the supplier's protocol (Invitrogen, Carlsbad, USA) in serum-free medium containing plasmid DNA. After $3 \mathrm{~h}$, serum-containing medium was added and the cells were incubated overnight. Finally, the transfection agent was replaced with medium containing $10 \%$ fetal bovine serum. Cells were fixed in $4 \%$ paraformaldehyde (PFA) in phosphate-buffered saline (PBS) for $15 \mathrm{~min}$ and covered with Vectashield mounting medium at the indicated time points (see results section). The range of transfection efficiency, as estimated by fluorescence microscopy, was approximately $20-30 \%$.

Cell death assay. Nuclei of fixed cells were DAPI stained and scored with a microscope (Zeiss Axiovert) as normal or apoptotic (i.e., pyknotic or fragmented nuclei). More than 200 transfected cells from at least three independent experiments per condition were scored and values were calculated as percentage of cells with apoptotic nuclei compared with the total cell count.

Fluorescence-based folding assay. Cells (CSM14.1 cells, see above) were cotransfected with hemagglutinin (HA)-tagged cdYFP expression vector, and respective pJRed-SOD1 expression vectors and were allowed to express the proteins overnight (see section Cell culture and transfection). Cells were fixed with $4 \%$ PFA in PBS for 10 min, washed three times with PBS, and quenched for 10 min with $50 \mathrm{mM}$ Tris $(\mathrm{pH} 7.5)-100 \mathrm{mM} \mathrm{NaCl}$. For the quantitation of folding efficiency, cells expressing HA-cdYFP were subjected to anti-HA antibody immunofluorescence analysis after permeabilization of fixed cells with $0.1 \%$ Triton X-100 in PBS for 20 min. Cells were blocked with $5 \%$ bovine serum albumin in PBS before incubation for $2 \mathrm{~h}$ with a 1:1000 dilution of the primary anti-HA monoclonal antibody (Covance Research Products, Columbia, MO) and subsequent incubation for $1 \mathrm{~h}$ with a 1:300 dilution of the Cy5-labeled goat antimouse secondary antibody (Jackson Laboratories, Bar Harbor, Maine). Cells were mounted in Mowiol. Three 5-min washes with PBS were performed between the steps.

Detection of YFP and Cy5 fluorescence, image acquisition, and image arithmetic were performed as previously described. ${ }^{21}$ Folding ratio frequency histograms were analyzed with the Igor Pro analysis package (Wavemetrics Inc., Portland, OR). Individual histograms were summed and cumulative histograms were normalized to the integrated counts, that is, to the number of observed pixels per experimental data set. For further analysis, see section Statistical analysis of FRET/folding efficiency distributions below.

Proteasome assay. The Pd2ECFP-N1 expression vector (ECFP-PEST vector) was purchased from Clontech (Mountain View, CA, USA). In this construct, residues $422-461$ of mouse ornithine decarboxylase (MODC) are fused to the C-terminus of ECFP. ${ }^{26}$ This region contains a PEST amino-acid sequence that targets the protein for degradation and results in rapid protein turnover. ECFP-PEST has a half-life of approximately $2 h{ }^{26}$ In our experiments, the ECFP-PEST vector was cotransfected with a conventional EYFP expression vector to allow normalization of ECFP-PEST fluorescence to concentration differences within (i.e., compartmentalization) and between (i.e., transfection efficiency) cells. The image intensity values were corrected for the laser power setting needed to obtain proper photon statistics under the various conditions. The linear calibration of the laser power was verified by comparison of the fluorescence intensities of fluorescent beads. The ratio images were masked by thresholding the fluorescence intensities in the CFP or YFP channel to remove noise in the non-cellular 
background. Similar to the folding assay using cdYFP, the proteasomal function, that is, normalized ECFP efficiency, was retrieved by simple image arithmetic (ECFP fluorescence/YFP reference fluorescence) using ImageJ software (http:// rsb.info.nih.gov/ij/). The proteasomal function in cells coexpressing myc-SOD1 protein was then normalized to the cells expressing only ECFP and YFP (see Results), so that the effects on proteasomal function were expressed in units of native proteasomal function. Proteasomal function frequency histograms were obtained from masked images of 10 cells for each condition, unless otherwise indicated. Folding ratio frequency histograms were analyzed by the Igor Pro analysis package. Normalized cumulative histograms were obtained and treated as for the folding assay.

Fluorescence lifetime microscopy. Fluorescence time decays were resolved by time-correlated single-photon counting (TCSPC) using an SPC830 acquisition board (Becker \& Hickl, Berlin). Two-photon excitation of GFP was performed at $900 \mathrm{~nm}$ by a femtosecond mode-locked ( $76 \mathrm{MHz}$ repetition rate) Ti:Sapphire Mira900F laser pumped by a Verdi-V8 laser (both from Coherent, Santa Clara, USA). The laser beam was fed to the scanning head of a Leica TSC-SP2AOBS confocal microscope. Fluorescence was collected on a multichannel plate photomultiplier (R3809U-50; Hamamatsu Photonics, Hamamatsu City, Japan) through a bandpass GFP filter at $515 \pm 30 \mathrm{~nm}$ (EGFP-HQset by Analysentechnik, Lagenfeld, Germany). The fluorescence transients were acquired using SPCIMAGE software (Becker \& Hickl), exported, and analyzed using an in-house-developed MATLAB (MathWorks, Natick, MA) toolbox. Images were acquired in $512 \times 512$ pixel format, collecting in excess of 1000 photons per pixel in 5-10 min.

SOD1 ubiquitination was imaged in CSM14.1 cells expressing EGFP or EGFPwt/mtSOD1 (pcDNA3.1 expression vector; Clontech) and REACh-ubiquitin constructs. Binding of Hsp70 to wt/mtSOD1 was imaged in CSM14.1 cells expressing EGFP-wt/mtSOD1 and REACh-Hsp70 (see also section Expression vectors). Cells were transiently transfected and fixed with $4 \%$ PFA in PBS $18 \mathrm{~h}$ after transfection (see above).

Image processing. Histograms from (cdYFP) folding responses were further processed using the Igor Pro package. Control histograms for cells that did not express SOD1 proteins were used to rescale the ratio values to folding efficiencies, that is, the average ratio was set to 1 to represent the base folding efficiency in control cells. The folding efficiency distributions of individual cells were normalized to an integrated value of unity. Individual normalized histograms for all cells within one condition were cumulated and renormalized to unity to compensate for different sample sizes.

Images of the CFP-PEST degradation assay were processed using the same procedure.

Similarly, distribution histograms were obtained from lifetime images of SOD1 ubiquitination and Hsp70 interaction experiments. Here, the lifetimes $(\tau)$ were converted to FRET efficiencies by comparison with the EGFP lifetime of cells expressing EGFP-SOD1 in the absence of REACh-labeled interaction partner $\left(\tau_{\mathrm{r}}\right)$ according to: $F R E T=1-\left(\tau / \tau_{r}\right)$. Proteasomes were defined as peri-nuclear punctate structures with high FRET between EGFP-SOD1 and REACh-ubiquitin and were segmented for processing by thresholding of the FRET efficiencies.

\section{Statistical analysis of FRET/folding efficiency distributions. As} the normalized histograms represent probability distribution functions, they can be used to estimate the probability of obtaining significant FRET on a cell-by-cell basis. Comparison of these results within one sample then provides the statistical values (average \pm standard error of the mean) and significance.

The cumulative distribution of the FRET efficiencies of control cells expressing EGFP-SOD1 without coexpression of the REACh-labeled Hsp70 or ubiquitin were fitted with a Gaussian function to obtain the average plus three times standard deviation value $(3 \sigma)$, which amounted to $14 \%$ of FRET. Any values exceeding this threshold thus represent increases that significantly deviate from the control situation. The probability of obtaining significant FRET can be derived for each cell by integration of the normalized FRET distribution histograms above the $3 \sigma$ threshold. The results for all cells within one sample were subjected to one-way ANOVA with Tukey's post-testing to evaluate the statistical significance of the average results.

SOD1 activity assay. The SOD1 activity assay was performed as described previously. ${ }^{22}$ Briefly, CSM14.1 cells were lysed in $\mathrm{ddH}_{2} \mathrm{O}$ by three freeze-thaw cycles, and lysates were clarified by centrifugation at $16100 \times g$ for $10 \mathrm{~min}$ at $4^{\circ} \mathrm{C}$.
Samples ( $20 \mu \mathrm{g}$ per lane) were loaded in native buffer and separated using $7.5 \%$ non-denaturing polyacrylamide gels. Gels were then soaked in $2.45 \mathrm{mM}$ nitro blue tetrazolium (NBT) solution for $20 \mathrm{~min}$, followed by incubation in developer solution for $15 \mathrm{~min}$ ( $28 \mathrm{mM}$ TEMED, $28 \mu \mathrm{M}$ riboflavin, $36 \mathrm{mM} \mathrm{KH}_{2} \mathrm{PO}_{4}$ ) at room temperature in the dark. Gels were then illuminated for 15 min until sufficient contrast between achromatic zones (dismutase activity) and blue background was achieved. Human wtSOD1 protein purified from erythrocytes (Sigma-Aldrich, Cölbe, Germany) was used as positive control.

AMS reaction disulfide assay. The thiol-specific reagent 4-acetamide-4'maleimidylstilbene-2,2'-disulfonic acid (AMS) was used to determine the extent of disulfide bond formation in SOD1-EGFP, following the protocol described by Jensen and Culotta, ${ }^{37}$ with minor variations. Briefly, cells expressing SOD1-EGFP were lysed in a buffer containing $6 \mathrm{M}$ guanidine- $\mathrm{HCl}, 50 \mathrm{mM}$ Tris $\mathrm{pH}$ 8.3, $3 \mathrm{mM}$ EDTA, $1 \mathrm{mM}$ bathocuproin sulfonate (BCS), and $0.5 \%$ Triton X-100. Lysates were sonicated and centrifuged to remove cellular debris. The supernatants were incubated in the presence of $1 \mathrm{mM}$ dithiothreitol (DTT) for $30 \mathrm{~min}$ (to reduce disulfide bonds), followed by incubation with $15 \mathrm{mM} \mathrm{AMS}$ for $60 \mathrm{~min}$ at $37^{\circ} \mathrm{C}$, or incubated only with AMS under oxygen-free conditions. Protein lysates were precipitated to remove unreacted DTT and AMS, solubilized in loading buffer without reducing agents, and subjected to SDS-PAGE on a gradient gel.

Western blot analysis. Western blot analysis was performed using EGFPSOD1-transfected CSM14.1 cells homogenized in lysis buffer containing $50 \mathrm{mM}$ Tris-HCl; $150 \mathrm{mM} \mathrm{NaCl} ; 1 \%$ Triton-X 100; $0.1 \mathrm{mM} \mathrm{PMSF}$; and $2 \mathrm{I} \mu / \mathrm{ml}$ pepstatin, leupeptin, and aprotinin, $\mathrm{pH}$ 8.3. Lysates were centrifuged at $16100 \times g$ and the protein concentration of the supernatant was determined using the BCA reagent (Pierce, Rockford, IL). After separating the lysates by reducing SDS-PAGE (10\% gel, $20 \mathrm{~g}$ protein per lane), proteins were transferred to polyvinylidene difluoride (PVDF) membrane and blocked in 5\% skimmed milk in PBS-T (0.1\% Tween 20$)$. Proteins were detected by incubation with primary antibodies directed against SOD1 (SOD-100; Stressgen, Victoria, Canada). Primary antibody was visualized by incubation with respective HRP-conjugated secondary antibodies (Dianova, Hamburg, Germany). Lysates from at least three different experiments were tested.

Statistical analysis. All values are expressed as mean \pm S.E.M. ANOVA followed by Tukey's post-testing was used to determine significance, unless otherwise indicated (NCSS Software; NCSS, Kaysville, USA).

Acknowledgements. We thank Christine Poser for excellent technical assistance, and Johannes Schlachetzki for critically reading the manuscript. This study was supported by the NeuroNE Network of Excellence within the 6th framework program (European Union grant LSHM-CT-2004-512039), by an EC fellowship for Neuroscience Early Stage Research Training to KS (NEUREST), by the Bundesminiterium für Bildung und Forschung (BMBF) on the FLI-CAM project within the Biophotonics III program, and by the DFG Cluster of Excellence (COE171) 'Microscopy at the Nanometer Range' with the Centre for Molecular Physiology of the Brain (CMPB) to FSW.

1. Rowland LP, Shneider NA. Amyotrophic lateral sclerosis. N Engl J Med 2001; 344 1688-1700.

2. Rabizadeh S, Gralla EB, Borchelt DR, Gwinn R, Valentine JS, Sisodia S et al. Mutations associated with amyotrophic lateral sclerosis convert superoxide dismutase from an antiapoptotic gene to a proapoptotic gene: studies in yeast and neural cells. Proc Natl Acad Sci USA 1995; 92: 3024-3028.

3. Bowling AC, Barkowski EE, McKenna-Yasek D, Sapp P, Horvitz HR, Beal MF et al. Superoxide dismutase concentration and activity in familial amyotrophic lateral sclerosis. J Neurochem 1995; 64: 2366-2369.

4. Bruijn LI, Houseweart MK, Kato S, Anderson KL, Anderson SD, Ohama E et al. Aggregation and motor neuron toxicity of an ALS-linked SOD1 mutant independent from wild-type SOD1. Science 1998; 281: 1851-1854.

5. Shinder GA, Lacourse MC, Minotti S, Durham HD. Mutant Cu/Zn-superoxide dismutase proteins have altered solubility and interact with heat shock/stress proteins in models of amyotrophic lateral sclerosis. J Biol Chem 2001; 276: 12791-12796.

6. Bruening W, Roy J, Giasson B, Figlewicz DA, Mushynski WE, Durham HD. Upregulation of protein chaperones preserves viability of cells expressing toxic $\mathrm{Cu} / \mathrm{Zn}$-superoxide dismutase mutants associated with amyotrophic lateral sclerosis. J Neurochem 1999; 72: 693-699 
7. McClellan AJ, Tam S, Kaganovich D, Frydman J. Protein quality control: chaperones culling corrupt conformations. Nat Cell Biol 2005; 7: 736-741.

8. Matsumoto G, Stojanovic A, Holmberg Cl, Kim S, Morimoto RI. Structural properties and neuronal toxicity of amyotrophic lateral sclerosis-associated $\mathrm{Cu} / \mathrm{Zn}$ superoxide dismutase 1 aggregates. J Cell Biol 2005; 171: 75-85

9. Bruijn LI, Becher MW, Lee MK, Anderson KL, Jenkins NA, Copeland NG et al. ALS-linked SOD1 mutant G85R mediates damage to astrocytes and promotes rapidly progressive disease with SOD1-containing inclusions. Neuron 1997; 18: 327-338.

10. Watanabe M, Dykes-Hoberg M, Culotta VC, Price DL, Wong PC, Rothstein JD. Histological evidence of protein aggregation in mutant SOD1 transgenic mice and in amyotrophic lateral sclerosis neural tissues. Neurobiol Dis 2001; 8: 933-941.

11. Tummala $H$, Jung $C$, Tiwari A, Higgins CM, Hayward LJ, Xu Z. Inhibition of chaperone activity is a shared property of several $\mathrm{Cu}, \mathrm{Zn}$-superoxide dismutase mutants that cause amyotrophic lateral sclerosis. J Biol Chem 2005; 280: 17725-17731.

12. Takeuchi H, Kobayashi $Y$, Yoshihara T, Niwa J, Doyu M, Ohtsuka K et al. Hsp70 and Hsp40 improve neurite outgrowth and suppress intracytoplasmic aggregate formation in cultured neuronal cells expressing mutant SOD1. Brain Res 2002; 949: 11-22.

13. Liu J, Shinobu LA, Ward CM, Young D, Cleveland DW. Elevation of the Hsp70 chaperone does not effect toxicity in mouse models of familial amyotrophic lateral sclerosis. J Neurochem 2005; 93: 875-882.

14. Kieran D, Kalmar B, Dick JR, Riddoch-Contreras J, Burnstock G, Greensmith L. Treatment with arimoclomol, a coinducer of heat shock proteins, delays disease progression in ALS mice. Nat Med 2004; 10: 402-405.

15. Cheroni C, Peviani M, Cascio P, Debiasi S, Monti C, Bendotti C. Accumulation of human SOD1 and ubiquitinated deposits in the spinal cord of SOD1G93A mice during moto neuron disease progression correlates with a decrease of proteasome. Neurobiol Dis 2005 18: $509-522$

16. Kabuta T, Suzuki Y, Wada K. Degradation of amyotrophic lateral sclerosis-linked mutant $\mathrm{Cu}, \mathrm{Zn}$-superoxide dismutase proteins by macroautophagy and the proteasome. J Biol Chem 2006; 281: 30524-30533.

17. Urushitani M, Kurisu J, Tsukita K, Takahashi R. Proteasomal inhibition by misfolded mutan superoxide dismutase 1 induces selective motor neuron death in familial amyotrophic lateral sclerosis. J Neurochem 2002; 83: 1030-1042.

18. Urushitani M, Kurisu J, Tateno M, Hatakeyama S, Nakayama K, Kato $\mathrm{S}$ et al. CHIP promotes proteasomal degradation of familial ALS-linked mutant SOD1 by ubiquitinating Hsp/Hsc70. J Neurochem 2004; 90: 231-244.

19. Kabashi E, Agar JN, Taylor DM, Minotti S, Durham HD. Focal dysfunction of the proteasome: a pathogenic factor in a mouse model of amyotrophic lateral sclerosis $J$ Neurochem 2004; 89: 1325-1335.

20. Ganesan S, Ameer-Beg SM, Ng TT, Vojnovic B, Wouters FS. A dark yellow fluorescent protein (YFP)-based resonance energy-accepting chromoprotein (REACh) for Forste resonance energy transfer with GFP. Proc Natl Acad Sci USA 2006; 103: 4089-4094.

21. Liman J, Ganesan S, Dohm CP, Krajewski S, Reed JC, Bähr M et al. Interaction of BAG and Hsp70 mediates neuroprotectivity and increases chaperone activity. Mol Cell Bio 2005: 25: 3715-3725.
22. Beauchamp C, Fridovich I. Superoxide dismutase: improved assays and an assay applicable to acrylamide gels. Anal Biochem 1971; 44: 276-287.

23. Hayward LJ, Rodriguez JA, Kim JW, Tiwari A, Goto JJ, Cabelli DE et al. Decreased metallation and activity in subsets of mutant superoxide dismutases associated with familial amyotrophic lateral sclerosis. J Biol Chem 2002; 277: 15923-15931.

24. Furukawa Y, Torres AS, O'Halloran TV. Oxygen-induced maturation of SOD1: a key role for disulfide formation by the copper chaperone CCS. EMBO J 2004; 23: 2872-2881.

25. Liu R, Li B, Flanagan SW, Oberley LW, Gozal D, Qiu M. Increased mitochondrial antioxidative activity or decreased oxygen free radical propagation prevent mutant SOD1mediated motor neuron cell death and increase amyotrophic lateral sclerosis-like transgenic mouse survival. J Neurochem 2002; 80: 488-500.

26. Li $X$, Zhao X, Fang $Y$, Jiang $X$, Duong $T$, Fan $C$ et al. Generation of destabilized green fluorescent protein as a transcription reporter. J Biol Chem 1998; 273: 34970-34975

27. Niwa J, Ishigaki S, Hishikawa N, Yamamoto M, Doyu M, Murata S et al. Dorfin ubiquitylates mutant SOD1 and prevents mutant SOD1-mediated neurotoxicity. J Biol Chem 2002; 277: 36793-36798.

28. Miyazaki K, Fujita T, Ozaki T, Kato C, Kurose Y, Sakamoto M et al. NEDL1, a novel ubiquitin-protein isopeptide ligase for dishevelled-1, targets mutant superoxide dismutase1. J Biol Chem 2004; 279: 11327-11335.

29. Di Noto L, Whitson LJ, Cao X, Hart PJ, Levine RL. Proteasomal degradation of mutant superoxide dismutases linked to amyotrophic lateral sclerosis. J Biol Chem 2005; 280: 39907-39913.

30. Lee HJ, Lee SJ. Characterization of cytoplasmic alpha-synuclein aggregates. Fibril formation is tightly linked to the inclusion-forming process in cells. J Biol Chem 2002; 277: 48976-48983.

31. Manzerra P, Brown IR. Expression of heat shock genes (hsp70) in the rabbit spinal cord: localization of constitutive and hyperthermia-inducible mRNA species. J Neurosci Res 1992; 31: 606-615

32. Morrison-Bogorad M, Pardue S, Mclntire DD, Miller EK. Cell size and the heat-shock response in rat brain. J Neurochem 1994; 63: 857-867.

33. Clement AM, Nguyen MD, Roberts EA, Garcia ML, Boillee S, Rule M et al. Wild-type nonneuronal cells extend survival of SOD1 mutant motor neurons in ALS mice. Science 2003; 302: 113-117.

34. Boillee S, Yamanaka K, Lobsiger CS, Copeland NG, Jenkins NA, Kassiotis G et al. Onset and progression in inherited ALS determined by motor neurons and microglia. Science 2006; 312: 1389-1392.

35. Turner BJ, Atkin JD, Farg MA, Zang DW, Rembach A, Lopes EC et al. Impaired extracellular secretion of mutant superoxide dismutase 1 associates with neurotoxicity in familial amyotrophic lateral sclerosis. J Neurosci 2005; 25: 108-117.

36. Haas SJ, Wree A. Dopaminergic differentiation of the Nurr1-expressing immortalized mesencephalic cell line CSM14.1 in vitro. J Anat 2002; 201: 61-69.

37. Jensen LT, Culotta VC. Activation of CuZn superoxide dismutases from Caenorhabditis elegans does not require the copper chaperone CCS. J Biol Chem 2005; 280: 41373-41379.

Supplementary Information accompanies the paper on Cell Death and Differentiation website (http://www.nature.com/cdd) 